\title{
L'ALTA BUROCRAZIA MINISTERIALE: MODELLI DI RECLUTAMENTO E CARRIERA
}

\author{
di Rosalba Chiarini
}

Il problema e le ipotesi

Il rendimento e la ricettività dei sistemi politici democratici in termini di capacità di risoluzione dei problemi da parte di apparati e strutture amministrative sono stati spesso interpretati facendo riferimento a caratteristiche culturali, politico-partitiche, socio-economiche ${ }^{1}$. L'adozione di diverse angolazioni analitiche di questo tipo ha senza dubbio contribuito ad accrescere la conoscenza degli apparati pubblici e del loro grado di funzionalità. Tuttavia, sembra produttivo insistere nell'analisi delle dimensioni organizzative delle burocrazie pubbliche, in particolare dei fattori attinenti al reclutamento e alla socializzazione in quanto, ormai da tempo, hanno dimostrato la loro importanza al fine di interpretare il comportamento di un'organizzazione anche nelle sue relazioni con l'ambiente esterno.

Le ricerche sulle élites amministrative condotte secondo questa prospettiva ${ }^{2}$ hanno in effetti registrato consistenti diso-

Questa ricerca è stata condotta nell'ambito di un progetto finanziato dal CNR (contratto n. 901239 del 1990). La ricerca è stata condotta su un campione costituito da soggetti appartenenti alle tre qualifiche dirigenziali - prima dirigenza, dirigenza superiore, dirigenza generale - ma il contributo di questo articolo è circoscritto alla dirigenza generale.

Ringrazio per i preziosi suggerimenti i Proff. Giorgio Freddi e Carlo Guarnieri. Desidero inoltre ringraziare Michele Sapignoli per l'aiuto nell'elaborazione dei dati e tutti coloro che, e sono molti, nelle amministrazioni centrali dello Stato mi hanno riservato collaborazione ed attenzione.

${ }^{1}$ A questo riguardo esempi significativi della prima prospettiva ci sono forniti da Putnam (1973) e da Aberbach, Putnam e Rockman (1981). La rilevanza dei fattori partitici e istituzionali è stata richiamata da Panebianco (1988). La prospettiva che ha sottolineato l'importanza delle variabili socio-economiche ci ha fornito studi ed elaborazioni sulle trasformazioni qualitative e quantitative delle burocrazie pubbliche. Sotto questo profilo, importanti sono i contributi di Rose (1976), Ridley (1979), Self (1972), Mayntz (1978) e, per il caso italiano, il contributo di Cassese (1983).

${ }^{2}$ Fra gli studiosi che si sono occupati dell'incidenza dei modelli di reclutamento e

RIVISTA ITALIANA DI SCIENZA POLITICA / a. XXV, n. 1, aprile 1995 
mogeneità sotto il profilo dello status e dell'ethos burocratico ${ }^{3}$, dell'incidenza politica della burocrazia e quindi dei rapporti con le istituzioni politiche di vertice, del grado di permeabilità dell'élite amministrativa, delle strategie di controllo sulla burocrazia adottate dalla classe politico-elettiva.

Sembra allora plausibile che configurazioni diverse di proprietà organizzative rilevanti - quali le modalità di reclutamento e carriera - possano in vario grado incidere sul contesto istituzionale rafforzandone $\mathrm{o}$, viceversa, indebolendone la capacità di rendimento. Da questo punto di vista, il caso italiano offre interessanti possibilità di approfondimento visto che la letteratura in materia, pur avendo in più occasioni sottolineato l'importanza delle modalità di reclutamento, socializzazione e carriera (Mortara 1989; Cassese 1985; Taradel 1989; Guarnieri 1988; Zan 1989) ha riservato scarso spazio alle ripercussioni che tali proprietà organizzative provocano sulla forza dell'istituzione. Si tratta allora di approfondire tali aspetti attraverso una duplice modalità: da un lato effettuando una ricerca empirica capace di evidenziare quei caratteri dell'élite forgiati dai meccanismi di reclutamento e carriera e, dall'altro lato, accertando la forza delle istituzioni amministrative espressa dal grado di controllo di importanti risorse organizzative.

A tal proposito questa ricerca si concentra su tre ministeri esemplificativi degli apparati pubblici centrali e marcati da dif-

carriera, ma non solo, sulle caratteristiche delle élites amministrative vedi soprattutto gli studi in chiave comparata di Aberbach, Putnam e Rockman (1981), Freddi (1968, 1982 e 1989), Page (1985), Peters (1987), Ridley (1983), Self (1972).

${ }^{3}$ Prestigio sociale e senso di appartenenza ad un gruppo selezionato non sembrano associati all'estrazione sociale, bensì a fattori organizzativi quali le modalità di reclutamento, socializzazione e avanzamento in carriera. Semplificando una realtà invece ricca di differenziazioni si possono, in generale, delineare due modelli polari di reclutamento e socializzazione. Da un lato, ci sono i sistemi basati su concorsi aperti a tutti i candidati in possesso di specifici requisiti (caso italiano), dall'altro lato ci sono sistemi di ingresso presidiati da istituzioni governative impegnate nella preparazione specialistica dell'alta dirigenza (caso francese). Altri sistemi amministrativi combinano un reclutamento per concorso con periodi di socializzazione presso istituzioni educative (caso tedesco e inglese). Questi ultimi, ma soprattutto un sistema come quello francese, agiscono favorevolmente sullo spirito di corpo e sul prestigio sociale. Allo stesso modo, regole che disciplinano l'avanzamento nella carriera basate su criteri di anzianità e automaticità della progressione hanno un effetto deprimente sullo spirito di corpo e sull'auto-stima dei burocrati, mentre sistemi di promozione interna selettivi e fondati sul criterio del merito li accentuano (Panebianco 1986). Per la sua attinenza con il sistema premi-punizioni di un'organizzazione, ovvero con quel sistema deputato a sanzionare, positivamente o negativamente, le prestazioni dei singoli al fine di garantire il rispetto dei comportamenti organizzativi attesi, è questo un aspetto estremamente importante del governo del personale (Zan 1989). 
ferenti modalità di reclutamento e carriera. La scelta - caduta sul Ministero dell'interno (MI), sul Ministero dell'industria, commercio e artigianato (MICA) e sul Ministero del lavoro e della previdenza sociale (MLPS) ${ }^{4}$ - è stata indirizzata da una strategia di indagine comparata di casi contrastanti quanto ai sistemi di reclutamento, socializzazione e carriera in corrispondenza alle finalità e ai compiti assegnati all'azione amministrativa. Da questo punto di vista i ministeri prescelti esemplificano l'evoluzione e la differenziazione dei compiti che di volta in volta lo Stato ha assunto nel corso dell'evoluzione delle sue funzioni. Il Ministero dell'interno rappresenta un caso emblematico di apparato pubblico tradizionale il cui imperativo è funzionale alla salvaguardia dei diritti fondamentali dei cittadini attraverso la tutela dell'ordine pubblico. Viceversa, il Ministero dell'industria, commercio e artigianato e il Ministero del lavoro e previdenza sociale rappresentano casi esemplificativi di apparati pubblici preposti all'esercizio di funzioni recentemente assunte dal sistema politicos.

Sulla base delle conoscenze messe a disposizione dalla letteratura organizzativa e politologica ci si aspetta che fra caratteristiche delle élites e caratteristiche delle organizzazioni ministeriali ci sia una congruenza tale da indurre a supporre che un forte governo del personale sia correlato ad una elevata unità e compattezza dell'istituzione amministrativa e, al contrario, che un debole governo del personale sia associato ad un basso grado di unità e compattezza dell'organizzazione. La letteratura ${ }^{6}$

${ }^{4} \mathrm{E}$ noto infatti che i prefetti dell'amministrazione civile del MI hanno uno stato giuridico particolare in conseguenza della funzione di rappresentanza del potere esecutivo. Il regime speciale per i prefetti ha nei contenuti propri degli istituti della disposizione, della aspettativa, della disponibilità e del collocamento a riposo per ragioni di servizio, quattro espressioni di differenziazione rispetto a quelli previsti per la generalità degli impiegati pubblici. Il dato curioso però è che la specialità è estesa a tutta la categoria dei prefetti, non solo a coloro che ricoprono l'incarico di capo di uffici periferici di prefettura (Casula 1972). Inoltre presso il MI è stata mantenuta la «carriera prefettizia» e il relativo criterio di avanzamento - il merito comparativo (vedi legge 340/1982). Preciso che presso gli altri due ministeri l'ordinamento del personale in carriere è stato abolito dalla legge 312/1980 e che non si riscontrano condizioni di diversità attinenti alle disposizioni normative che regolano l'ingresso nella dirigenza e i criteri di avanzamento.

${ }^{5} \mathrm{Ci}$ si riferisce a quelle funzioni di intervento socio-economico e di intermediazione di interessi diversi e spesso confliggenti che caratterizzano gli attuali regimi liberaldemocratici (Freddi 1986, 1989).

${ }^{6} \mathrm{Ci}$ si riferisce a quella letteratura che ha stabilito un nesso fra il concetto di potere e il concetto di scambio (Emerson 1962; Blau 1964) e che ha collegato i rapporti di potere ad una diseguale capacità di controllo delle aree di incertezza organizzativa 
che ispira tale ipotesi vede nel comportamento di un'organizzazione l'esito dell'attività di controllo delle principali zone di incertezza per l'organizzazione. È ragionevole considerare l'attività di reclutamento, socializzazione e carriera una delle attività cruciali da cui dipende il funzionamento dell'organizzazione; altrettanto plausibile è ipotizzare che la capacità di controllo di queste e di altre variabili organizzative rappresenti una risorsa anche ai fini dell'acquisizione di un peso specifico nell'ambito dei rapporti tra organizzazioni. Relativamente alle istituzioni amministrative, ciò induce a ritenere che la diversa configurazione delle proprietà organizzative e il differente grado di governo delle stesse abbia conseguenze in termini di strategie di controllo sulla burocrazia da parte degli attori politico-elettivi.

Concludo fornendo qualche informazione sui due livelli di articolazione della ricerca. Il primo livello dell'indagine empirica - volto a rilevare il risultato della differenziazione dell'élite sotto l'influenza dei modelli di reclutamento e carriera - riguarderà un campione di dirigenti generali le cui caratteristiche riveleranno la minore o maggiore capacità di controllo dell'istituzione di queste importanti dimensioni. Il secondo livello tenterà di mettere in luce la reciproca associazione fra assetto del personale e capacità di controllo da parte dell'istituzione amministrativa di altre importanti fonti di incertezza quali la struttura gerarchica, l'assegnazione di personale, il funzionamento degli uffici, le regole per disciplinare l'accesso di soggetti esterni.

Nelle prossime tre sezioni specifico analiticamente ed empiricamente le due tappe attraverso le quali si articola lo studio dei tre ministeri, mentre affido alla sezione conclusiva alcune considerazioni sul rapporto tra burocrazia e politica.

\section{Modelli di reclutamento, socializzazione e carriera}

Per studiare l'assetto organizzativo dei tre ministeri sembrano cruciali tre fattori: il reclutamento, la socializzazione e la carriera. Il reclutamento sta ad indicare il processo di selezione

(Crozier 1963; Crozier e Friedberg 1977). In particolare, questi ultimi autori hanno individuato le principali fonti di incertezza di un'organizzazione nella competenza, nei rapporti tra l'organizzazione e l'ambiente, nelle informazioni, nelle regole organizzative. Per un'illustrazione di questa letteratura vedi Panebianco (1989). 
di individui che andranno a far parte di un'organizzazione. Con l'espressione socializzazione organizzativa si intende il processo attraverso il quale l'organizzazione promuove una condivisione di valori da parte dei membri, tale da ispirare le azioni individuali in ambito organizzativo. Con il termine carriera si intende «quella trafila di posizioni attraverso le quali si raggiungono posizioni di livello più elevato. Essa è il prodotto della struttura di opportunità e dei meccanismi selettivi che ai diversi livelli aprono o chiudono l'accesso agli elementi nuovi» (Cotta 1979, 43).

L'analisi delle caratteristiche dell'alta dirigenza presterà attenzione sia al $c h i$ viene reclutato, sia al come avviene il reclutamento. A questo scopo saranno esaminati i fattori socio-anagrafici - sesso, età, provenienza territoriale - e le modalità di promozione, carriera e ingresso nella qualifica di dirigente generale di un campione di soggetti incardinati nei ruoli dell'alta dirigenza ministeriale. Di conseguenza, si cercherà di determinare il ruolo che determinate posizioni, incarichi, competenze svolgono nel determinare uno dei seguenti itinerari:

- l'ascesa nella carriera ministeriale segnalata dal passaggio, all'interno di un ministero, da una posizione sotto-ordinata alla posizione di dirigenza generale;

- la cooptazione dall'esterno caratterizzata dal passaggio da arene sociali ed economiche esterne ad un ministero;

- il transito attraverso il ministero, modalità attraverso la quale si realizzano contemporaneamente la nomina a dirigente generale di un soggetto esterno e la sua destinazione ad altre strutture pubbliche attraverso il collocamento in fuori ruolo?

L'analisi delle caratteristiche della carriera del soggetto prima dell'ingresso nel ruolo dell'alta burocrazia consentirà di identificare differenti tipi di mobilità che in base alle indicazioni della letteratura sembrano rispondere: a) al tipo di socializzazione che caratterizza il sistema burocratico; $b$ ) al grado di controllo esercitato dal superiore.

Secondo la prima impostazione una elevata mobilità indica una socializzazione rivolta a plasmare in senso cosmopolita il futuro dirigente; viceversa, una bassa mobilità rivela una socia-

\footnotetext{
${ }^{7}$ L'istituto del collocamento fuori ruolo si configura «quando l'impiegato, destinato a prestare servizio presso altra amministrazione, viene posto fuori dai quadri organici della sua amministrazione, con la conseguenza che il posto può essere ricoperto da altro titolare» (Virga 1973, 651).
} 
lizzazione localista ${ }^{8}$. Per la seconda impostazione, un'accentuata mobilità segnala una elevata ricettività del soggetto nei confronti delle istanze manifestate dal superiore, mentre una mobilità ridotta individua un burocrate meno ricettivo'. I casi polari sono evidentemente rappresentati da una mobilità intra-organizzativa e contenuta e da una mobilità inter-organizzativa ed elevata ${ }^{10}$.

Non vi è dubbio che un altro aspetto saliente in stretta connessione con le dinamiche di dispiegamento della carriera è la partecipazione dell'alta burocrazia ai processi decisionali. Molte ricerche hanno dimostrato che l'attività amministrativa si accompagna ad un certo margine di discrezionalità che dota la burocrazia di un potere decisionale autonomo, ma soprattutto che l'ambito decisionale ha dilatato enormemente i suoi confini con il passaggio dai regimi oligarchico-liberali ai regimi di democrazia di massa. Ciò ha comportato l'assunzione, da parte delle strutture burocratiche, di funzioni di intervento economico-sociale e funzioni di intermediazione fra interessi organizzati, che si sono aggiunte a quelle tradizionali di regolamento e controllo (Freddi 1982 e 1986). La partecipazione della burocrazia alla formazione delle decisioni autoritative ha allora almeno tre implicazioni: 1) la burocrazia partecipa a funzioni istituzionalmente assegnate ad altre strutture del sistema politico; 2) attraverso questo processo la burocrazia influenza i contenuti delle attività e la dinamica complessiva del sistema politico; 3) la burocrazia si trova a svolgere un ruolo importante nel processo di

${ }^{8}$ Il richiamo è ai contributi di Merton (1949) e Gouldner (1957). Gli autori hanno ipotizzato identità organizzative «locali» o «cosmopolite» sulla base, rispettivamente, della identificazione univoca con i valori tradizionali dell'istituzione (local role), o viceversa, della identificazione con i valori socio-professionali di gruppi di riferimento (cosmopolitan role).

${ }^{9}$ Partendo dall'ipotesi che un dato modello di mobilità possa essere un indicatore del controllo esercitato dal superiore, McGregor (1974) stabilisce che una accentuata mobilità segnala una elevata ricettività del soggetto nei confronti delle istanze manifestate dal superiore, mentre una mobilità ridotta individua un burocrate meno ricettivo. I risultati della ricerca suggeriscono che i funzionari di successo nel sistema statunitense - esclusi i funzionari degli affari esteri - conquistano le più alte posizioni di influenza rimanendo ancorati ad un'unica agenzia o dipartimento.

${ }^{10}$ Esempio di una simile ricerca è costituito dai due modelli ideali di reclutamento individuati da Aberbach, Putnam e Rockman (1981) nello studio dei percorsi di carriera dei membri dell'élite governativa. Il guild system contempla un lungo apprendistato in una sola organizzazione e assicura una socializzazione intra-istituzionale dell'élite; l'entrepreneurial system risulta caratterizzato da ingresso laterale e provenienza da altre carriere e istituzioni e ricerca l'integrazione fra diverse élites. 
articolazione e aggregazione degli interessi e quindi nella trasmissione della domanda politica (Guarnieri 1977).

Il quadro appena tratteggiato schiude un ulteriore tema di indagine: quello della professionalità che contraddistingue l'azione della dirigenza ministeriale. L'analisi del contenuto delle mansioni accompagnata da quella della formazione e delle esperienze maturate dai dirigenti permetterà di riscontrare l'esplicarsi di capacità prevalentemente amministrative o prevalentemente politiche, tenendo conto di configurazioni intermedie in grado di dar conto della diversa combinazione degli elementi.

L'ipotesi di fondo è che tale professionalità sia influenzata da una serie di fattori: la qualifica ricoperta, l'appartenenza organizzativa, l'esperienza presso gli organi politici (segreterie particolari e gabinetti) degli apparati pubblici, il tipo di formazione, l'età.

Al fine di valutare complessivamente le variazioni delle modalità di reclutamento, socializzazione e carriera proprie di ogni ministero appare utile fissare due configurazioni speculari di proprietà analitiche rispettivamente denominate modello inside e modello outside ${ }^{11}$.

Il modello inside prevede le seguenti caratteristiche:

- un reclutamento sulla base di qualificazioni acquisite, in un arco di tempo piuttosto lungo, all'interno del sistema organizzativo di appartenenza (provenienza);

- un peso ridotto e non condizionante della partecipazione ad organi politici (incarico politico);

- una modalità di accesso all'alta dirigenza che esclude la cooptazione da una qualifica che non sia quella di dirigente superiore (accesso alla dirigenza);

- un processo di avanzamento attraverso tutte le posizioni verticali (progressione di qualifica);

- un elevato tasso di partecipazione ad organismi di carattere intraorganizzativo (incarichi);

${ }^{11}$ La definizione dei due modelli sintetici risente dello spunto che ho tratto da Ridley $(1983,180)$ il quale distingue fra due modelli alternativi: il service career-based e il service job-based. Il primo prevede che i funzionari siano reclutati in base a qualificazioni generaliste all'inizio della loro vita professionale e che dispieghino l'intera carriera nell'ambito di un'unica organizzazione attraverso promozioni interne che escludono la competizione con l'esterno. Il secondo modello prevede che i funzionari vengano reclutati per ricoprire specifici ruoli sulla base di qualificazioni attinenti all'incarico. Le loro prospettive di carriera risentono della competizione con gli outsiders. 
- la natura composita delle attività svolte durante la carriera (contenuto delle attività);

- il carattere prevalentemente giuridico della formazione (laurea);

- il settore operativo quale principale contesto di maturazione dell'esperienza lavorativa (settore);

- una mobilità piuttosto contenuta e di carattere intra-organizzativo (sede di servizio);

- un'età piuttosto avanzata (età).

Il modello outside, viceversa, si articola nelle seguenti proprietà:

- un reclutamento sulla base di qualificazioni acquisite all'esterno del sistema organizzativo di appartenenza (provenienza);

- un peso forte e condizionante della partecipazione ad organi politici (incarico politico);

- una modalità di accesso all'alta dirigenza che prevede la cooptazione da una qualifica inferiore e diversa da quella di dirigente superiore (accesso alla dirigenza);

- un processo di avanzamento che evita di attraversare tutte le posizioni verticali (progressione di qualifica);

- un elevato tasso di partecipazione ad organismi di carattere interorganizzativo (incarichi);

- la natura esclusiva delle attività svolte durante la carriera (contenuto delle attività);

- il carattere specialista della formazione (laurea);

- il settore di staff ${ }^{2}$ quale principale contesto di maturazione dell'esperienza lavorativa (settore);

- una mobilità piuttosto elevata e di carattere interorganizzativo (sede di servizio);

- un'età piuttosto giovane (età).

I due modelli sintetici sopra delineati servono a dare una caratterizzazione all'insieme dei dati che emergeranno dall'analisi empirica.

${ }^{12}$ Se unità di line sono le unità organizzative che svolgono un compito direttamente connesso ai fini organizzativi, unità di staff sono quelle unità il cui compito è strumentale rispetto al lavoro svolto dalla line. 


\section{I dirigenti generali: un'analisi empirica}

I dati che presenterò sono quelli di un campione di dirigenti generali ${ }^{13}$ inquadrati, rispettivamente, nei tre ministeri prescelti ${ }^{14}$.

Sulla base degli elenchi del personale, aggiornati al $1^{\circ}$ gennaio 1990, ho selezionato un campione di unità dell'Amministrazione Civile del MI dato l'elevato numero (198) di soggetti inquadrati nelle qualifiche di prefetto e prefetto di Ia classe. L'adozione di un rapporto di uno a dieci, ha condotto ad un campione di 20 unità. Presso il MICA e il MLPS sono state analizzate le intere popolazioni - rispettivamente 10 e 25 unità - data l'esiguità numerica. La tabella 1 riassume il risultato dell'intera operazione di selezione delle unità.

L'operazione successiva, vale a dire il rilevamento delle caratteristiche dei dirigenti generali, è stata condotta sia attraverso la consultazione di documenti in possesso dell'amministrazione, sia attraverso interviste.

Il resoconto dei dati emersi dalla ricerca partirà dall'esame di due proprietà - il sesso e la provenienza geografica - che sembrano influenzate da quelle caratteristiche del sistema socioeconomico che, per quanto riguarda il primo aspetto, frenano l'ingresso delle donne nell'alta burocrazia pur in presenza di un forte tasso di femminizzazione della pubblica amministrazione e, per quanto riguarda il secondo aspetto, incentivano i laureati meridionali ad orientarsi verso il pubblico impiego viste le scarse alternative offerte dal mercato del lavoro.

La prima proprietà - il sesso - mostra un risultato ormai consueto in quanto sufficientemente documentato da studi sul personale burocratico: tutti i dirigenti generali del MICA e del MLPS presenti nei ruoli del 1990 sono di sesso maschile. L'affermazione vale anche per i prefetti del MI, anche se i dati presentati riguardano solo un decimo della popolazione.

${ }^{13}$ La dirigenza dello Stato, in base al decreto 748/1972, si articola in tre qualifiche che, in ordine ascendente, sono la prima dirigenza, la dirigenza superiore e la dirigenza generale. A quest'ultima si accede in base alla nomina politica da parte del Consiglio dei ministri. Il decreto legislativo 29/1993 di riforma del pubblico impiego e i due successivi decreti 470 e 546 del 1993 hanno ridotto le qualifiche dirigenziali a due, ma la riforma ha appena preso avvio.

${ }^{14}$ A rigor di legge (vedi DPR 748/1972) non sarebbe corretto considerare i viceprefetti ispettori, $i$ viceprefetti ed i prefetti al pari di primi dirigenti, dirigenti superiori e dirigenti generali ma, al fine di procedere ad una comparazione sostanziale con il personale degli altri due ministeri, si procederà all'assimilazione delle qualifiche. 
TAB. 1. I casi selezionati per ministero

\begin{tabular}{lcc}
\hline & N. & $\%$ \\
\hline Ministero dell'interno & 20 & 36,4 \\
Ministero dell'industria, commercio e artigianato & 10 & 18,2 \\
Ministero del lavoro e della previdenza sociale & 25 & 45,5 \\
Totale & 55 & 100,0 \\
\hline
\end{tabular}

I dati attinenti alla provenienza geografica possono essere adeguatamente aggregati in base alla tripartizione del territorio nazionale individuata da Bagnasco (1977). Le tre formazioni territoriali, ognuna caratterizzata da peculiarità sociali ed economiche per tempi e modalità del processo di sviluppo, vengono rispettivamente denominate «centrale», «periferica», «marginale». La prima area è caratterizzata da centralità economica e presenza della grande impresa; l'economia dell'Italia «periferica» risulta basata sulla piccola e media impresa; l'Italia «marginale» è segnata dai caratteri del sottosviluppo. L'aggregazione individua: a) l'Italia del Nord-Ovest, costituita da Piemonte, Valle d'Aosta, Liguria e Lombardia; b) l'Italia periferica del Centro e del Nord-Est, con le tre Venezie, l'Emilia-Romagna, la Toscana, le Marche e l'Umbria; c) l'Italia marginale, comprendente il Lazio, le regioni meridionali e quelle insulari.

Siamo di fronte ad un altro risultato scontato che non evidenzia differenze significative fra i tre ministeri: la provenienza geografica della quasi totalità dei dirigenti analizzati è da ricondurre a zone marcate da sottosviluppo (tab. 2) ${ }^{15}$. Vanno notati però i quattro soggetti appartenenti al MI che, al contrario, pro-

\footnotetext{
${ }^{15}$ In merito alla provenienza territoriale dell'alta burocrazia, in Italia il rapporto alquanto lineare fra il processo di crescita burocratica e la meridionalizzazione dei quadri che si snoda a partire dal periodo post-unitario ha consentito di formulare l'ipotesi secondo la quale «l'accesso sempre più largo degli strati intellettuali meridionali (e specialmente dei laureati in giurisprudenza) nella carriera direttiva, serve ad assorbire un crescente malcontento di ceti medi e ad acquisirne il consenso. La meridionalizzazione dell'élite amministrativa, a sua volta, se serve alla «classe politica» - perché le consente di aprire un canale che assicura una certa mobilità a un segmento sociale altrimenti destinato a diventare opposizione, a causa delle condizioni di sottosviluppo dell'area di provenienza - provoca, però, uno scostamento tra i vertici della «classe amministrativa» e la dirigenza politica (...)» (Cassese 1977, 4).
} 
TAB. 2. Area territoriale di origine per ministero

\begin{tabular}{lrccc}
\hline & MI & MICA & MLPS & Totale \\
\hline Italia «centrale» & 1 & 0 & 0 & 1 \\
Italia «periferica» & 3 & 0 & 2 & 5 \\
Italia «marginale» & 16 & 10 & 23 & 49 \\
Totale & 20 & 10 & 25 & 55 \\
\hline
\end{tabular}

TAB. 3. L'estrazione organizzativa per ministero

\begin{tabular}{|c|c|c|c|c|c|c|c|}
\hline & \multirow{2}{*}{$\begin{array}{l}\text { MI } \\
\text { n. } \quad \%\end{array}$} & \multicolumn{2}{|c|}{ MICA } & \multicolumn{2}{|c|}{ MLPS } & \multicolumn{2}{|c|}{ Totale } \\
\hline & & & $\%$ & n. & $\%$ & & $\%$ \\
\hline burocrati di carriera & 20100,0 & 7 & 70,0 & 13 & 52,0 & 40 & 72,7 \\
\hline altra PA-ordinaria & 0 & 0 & & 6 & 24,0 & 6 & 10,9 \\
\hline PA-parallela & 0 & 3 & 30,0 & 3 & 12,0 & 6 & 10,9 \\
\hline arene sociali & 0 & 0 & & 3 & 12,0 & 3 & 5,5 \\
\hline Totale & 20 & 10 & & 25 & & 55 & 100,0 \\
\hline
\end{tabular}

vengono da zone economicamente non marginali e che sembrano rivelare motivazioni di scelta del pubblico impiego diverse rispetto a quelle abbondantemente messe in evidenza dalla letteratura ${ }^{16}$.

A questo punto illustro i dati relativi all'estrazione organizzativa (tab. 3). Un primo elemento da sottolineare è la differenziazione fra MI da un lato e MICA-MLPS dall'altro, in merito alla presenza di un gruppo di dirigenti generali che, precedentemente all'inserimento presso uno di questi due ministeri, ricopriva incarichi presso altre organizzazioni. Si tratta di 15 soggetti che provengono da altri ministeri (sei), da enti pubblici solitamente classificati nell'ambito del sistema amministrativo parallelo (sei), da organizzazioni di rappresentanza degli interessi (tre).

Il MICA prevede 10 posizioni di alta dirigenza amministrativa: tre di esse sono occupate da personale di estrazione extra-organizzativa. Più precisamente, tali soggetti provengono dal sistema pubblico parallelo e testimoniano lo stretto rapporto esistente fra

${ }^{16}$ Vedi la nota precedente. Motivazioni riconducibili alle scarse opportunità di impiego del mercato del lavoro meridionale sono addotte anche da Ammassari, Garzonio e Ferraresi (1969), Ferraresi (1980), Cerase (1990). 
il ministero e i grandi enti pubblici. Due di essi risultano insediati, in qualità di direttori generali, in due fra le più robuste direzioni (DG fonti di energia e DG produzione industriale) del ministero. Il restante $70 \%$ della alta dirigenza del MICA è costituito da personale di carriera sul quale si tornerà in seguito.

Più eterogenea, dal punto di vista della estrazione organizzativa, è la dirigenza generale del MLPS. I burocrati (tredici) che hanno trascorso la loro vita professionale all'interno del ministero sono affiancati da burocrati (sei) di carriera provenienti $\mathrm{da}$ altri ministeri, da personale (tre) proveniente da amministrazione locale, organizzazione internazionale, ente pubblico, e infine, da personale (tre) proveniente dalla CISL.

Anche se i risultati presentati non hanno considerato la dimensione temporale, $i$ dati raccolti hanno messo in luce la sostituzione del personale di carriera con personale non solo esterno, ma estraneo alla pubblica amministrazione, il cui comun denominatore è la frequentazione e la socializzazione politica. Solo 1'8\% dei dirigenti generali del MLPS risulta escluso da esperienze di carattere politico: il $92 \%$ della dirigenza ha ricoperto almeno un incarico presso la struttura di gabinetto o la segreteria particolare del ministro e/o del sottosegretario di stato. Sintomatici di un diverso modello di ingresso alla dirigenza generale sono i dati relativi al MICA e al MI, i quali esibiscono invece una percentuale di incarichi politici pari, rispettivamente, al $30 \%$ e al $25 \%$.

Tornando al tema della estrazione della dirigenza generale, il MI si distingue per la esclusività della provenienza interna: tutto il campione proviene dalla carriera prefettizia. Tale fenomeno sembra indicare che l'ingresso nel corpo dei prefetti rappresenta l'ultima tappa di un processo graduale che ha in precedenza previsto l'accesso alla qualifica di viceprefetto e, prima ancora, a quella di viceprefetto ispettore.

Ad una prima sintesi dei risultati emersi in merito a due variabili (provenienza, incarichi in organi politici), il MI si dimostra un ministero ad alta socializzazione intraorganizzativa: non ammette ingressi laterali e non subordina la nomina a prefetto alla vicinanza con il potere politico. Tale impressione è confermata dalle tabelle 4 e 5 che illustrano, rispettivamente, i dati di accesso alla prima dirigenza e alla dirigenza superiore. Il MI esclude salti di qualifica che configurino meccanismi di cooptazione: tutti i prefetti hanno maturato esperienza nelle precedenti qualifiche. 
TAB. 4. Accesso alla qualifica di vice prefetto ispettore/primo dirigente per ministero

\begin{tabular}{lrccc}
\hline & MI & MICA & MLPS & Totale \\
\hline no ingresso in PD & 0 & 4 & 12 & 16 \\
sì ingresso in PD & 20 & 6 & 13 & 39 \\
Totale & 20 & 10 & 25 & 55 \\
\hline
\end{tabular}

TAB. 5. Accesso alla qualifica di vice prefetto/dirigente superiore per ministero

\begin{tabular}{lcccc}
\hline & MI & MICA & MLPS & Totale \\
\hline no ingresso in DS & 0 & 8 & 18 & 26 \\
sì ingresso in DS & 20 & 2 & 7 & 29 \\
Totale & 20 & 10 & 25 & 55 \\
\hline
\end{tabular}

Un diverso stato di cose si presenta presso gli altri due ministeri. Al MICA, l' $80 \%$ della dirigenza generale è stato reclutato attraverso cooptazione o dal primo livello della dirigenza $(40 \%)$ o da ambienti esterni al ministero (40\%).

Presso il MLPS, il 48\% del totale dei dirigenti generali non ha ricoperto incarichi di prima dirigenza e il $72 \%$ non ha ricoperto le funzioni di dirigente superiore. Nel primo caso, la cooptazione è avvenuta da ambienti esterni, nel secondo, al $48 \%$ si aggiunge il $24 \%$ di coloro che sono cooptati dalla prima dirigenza.

Se il mancato ingresso nella «prima dirigenza» sembra rispondere unicamente a logiche di cooptazione, una spiegazione organizzativa può essere individuata per il mancato ingresso nella «dirigenza superiore» di molti dirigenti del MICA e del MLPS. L'analisi dei criteri di accesso alla «prima dirigenza» ${ }^{17} \mathrm{e}$

${ }^{17}$ In ordine all'accesso alla prima dirigenza, il DPR 748/1972 prevede l'innovativo sistema di reclutamento basato sul corso-concorso di formazione dirigenziale a cui attende la Scuola superiore di pubblica amministrazione. L'ammissione al corso si consegue mediante concorso per titoli di servizio, aperto a coloro che hanno maturato almeno 5 anni di servizio nella qualifica di direttore di sezione o equiparata. Il corso, della durata di 14 mesi, è ad indirizzo professionale, prevede esperienze presso aziende pubbliche e private ed esami finali. In realtà, fino al 1984, il sistema innovativo basato sul corso-concorso è rimasto bloccato. Il sistema transitorio, basato sullo scrutinio per merito comparativo riservato a funzionari dei ruoli ad esaurimento (legge 583/1978), è diventato permanente fino alla introduzione della legge 301/1984. Quest'ultima, oltre a prevedere il concorso pubblico, a cui possono partecipare sia esterni sia interni all'am- 
alla «dirigenza superiore» ${ }^{18}$ non ha, a questo fine, messo in luce implicazioni rilevanti diversamente dall'analisi delle funzioni dirigenziali associate alla dirigenza superiore che sembrano assumere un valore diverso a seconda del contesto ministeriale.

$\mathrm{Nel}$ caso del MI, gli incarichi di «vicario di prefettura» presso la sede provinciale e di «dirigente di servizio» presso il ministero, previsti dalla dirigenza superiore, rendono appetibile la qualifica. Sia la prefettura che il servizio ${ }^{19}$, per la loro natura operativa, rappresentano un contesto organizzativo premiante. Al contrario, presso gli altri due ministeri, l'incarico di vicedirettore, $\mathrm{di}$ ispettore generale e di consigliere ministeriale aggiunto, tutti previsti dalla dirigenza superiore, risultano privi di efficacia operativa (vedi line). L'affermazione può sembrare paradossale ma così non è: il vicedirettore ha una reale possibilità di dispiegare la propria autorità solo in assenza del direttore generale, eventualità poco frequente e non programmabile; l'azione dell'ispettore generale è povera di conseguenze dato che controllo e sanzioni (positive e negative) hanno tanto più efficacia se praticati dal diretto superiore gerarchico, non da individui esterni e lontani dalla line; il consigliere ministeriale aggiunto, infine, è un dirigente che non dirige. Le sue prerogative - lo studio e la ricerca - configurano un ruolo del tutto ambiguo, compresso fra quelli rispettivamente ricoperti dall'ufficio legislativo e dal gabinetto del ministro.

L'inquadramento all'interno della dirigenza superiore, pertanto denota una sottrazione di status rispetto al precedente inquadramento - la prima dirigenza - e rispetto a quello successivo - la dirigenza generale - a cui si risponde cercando di evitare la qualifica.

ministrazione, ripropone il corso-concorso di formazione dirigenziale - questa volta di 6 mesi - ma in alternativa ad altre due modalità (esame speciale e concorso pubblico) di ingresso nella prima dirigenza e questo consente, in ultima analisi, di evitarlo. Queste norme non riguardano il MI che ha conservato lo scrutinio per merito comparativo quale criterio di promozione e quindi di accesso alle qualifiche. Un'avvertenza è d'obbligo: l'assetto sopra descritto è stato recentemente riformato del decreto legislativo 29/1993 e dai due successivi decreti di correzione n. 470 e n. 546 del 1993.

${ }^{18}$ Per l'accesso alla dirigenza superiore il DPR 748/1972 dispone o il turno di anzianità o il concorso per titoli. Queste norme non riguardano il MI che ha conservato lo scrutinio per merito comparativo quale criterio di promozione dalla qualifica di viceprefetto ispettore a quella di viceprefetto. Anche in questo caso va ricordato l'intervento della normativa di riforma già menzionata nella nota precedente.

${ }^{19} \mathrm{Si}$ tratta di un livello organizzativo, presente solo presso il $\mathrm{MI}$, sopra-ordinato rispetto alle divisioni della direzione generale. 
Una sintesi, in chiave di socializzazione, in merito ad altre tre variabili (accesso alla prima dirigenza, accesso alla dirigenza superiore, incarico associato alla dirigenza superiore) permette di evidenziare la diversa importanza che le tappe formali di avanzamento (qualifiche) rivestono nei tre ministeri. La linea di differenziazione, anche in questo caso, distingue il MI dagli altri due ministeri. Il primo dimostra la forza della socializzazione intraorganizzativa assicurando il passaggio attraverso tutte le qualifiche dei futuri prefetti. A questo si accompagna l'esercizio di discrezionalità dettato dalla applicazione del merito comparativo quale criterio di avanzamento ${ }^{20}$, che può eventualmente accelerare o decelerare la progressione dei candidati. Il passaggio in tutte le qualifiche ${ }^{21}$, la natura operativa degli incarichi ricoperti, la doppia esperienza presso le strutture centrali del ministero e presso la prefettura, vanno interpretati come indicatori di una graduale formazione della professionalità del prefetto. $\mathrm{Al}$ contrario, i dirigenti generali degli altri due ministeri mostrano un iter marcato da un avanzamento in qualifiche non sequenziali.

Il numero di incarichi ricoperti da ogni soggetto in organi esterni (consigli di amministrazione di enti ed aziende pubbliche; collegi di revisori di conti; delegazioni all'estero) e in organi interni al ministero (commissioni tecniche) ci consente di valutare il tipo e il grado delle esperienze dei burocrati.

Leggendo i dati che documentano la presenza dell'alta dirigenza in seno ai consigli di amministrazione è facile notare che la partecipazione diminuisce man mano che si passa dal MICA $(100,0 \%)$, al MLPS $(52,0 \%)$, al MI (50\%). Naturalmente il numero di incarichi cresce con l'aumentare della qualifica.

Gli incarichi in organi di controllo manifestano una frequenza in questo ordine: il $72,0 \%$ per il MLPS, il $60 \%$ per il MICA, il 16,7\% per il MI.

Per quanto riguarda la partecipazione a delegazioni all'estero, la quale esprime un interesse del ministero ad intervenire in arene sovra-nazionali, $i$ dati mostrano una tendenza interessante

${ }^{20}$ Vedi note 17 e 18.

${ }^{21}$ C'è anche la prassi di conferire funzioni superiori al funzionario sottoponendolo ad una prova di abilità quasi assente presso gli altri due ministeri che quindi rinunciano a tale meccanismo di valutazione. Considerando la discrezionalità che la burocrazia del MI esercita sulle promozioni, anche l'attribuzione di mansioni superiori rappresenta una circostanza per sanzionare positivamente o negativamente il comportamento di un soggetto. 
$-\mathrm{MI}=100 \%, \mathrm{MICA}=40 \%$, MLPS $=28 \%-$ che rivela l'impegno dei prefetti in commissioni CEE impegnate spesso sui temi della sicurezza.

Gli incarichi di partecipazione a commissioni tecnico-consultive istituite presso $\mathrm{i}$ ministeri e formate da rappresentanti delle diverse associazioni interessate dalle politiche ministeriali, oltre che da rappresentanti dello stesso ministero, rivelano che la pratica è diffusa, in primo luogo, presso il MICA $(100,0 \%)$, poi al MI $(66,6 \%)$, e quindi al MLPS $(60 \%)$.

Il significato, in termini di socializzazione interna/esterna, $\mathrm{da}$ attribuire alle esperienze relative ai quattro tipi di incarico partecipazione a consigli di amministrazione, collegi di revisori, delegazioni all'estero, commissioni tecniche - deve essere piuttosto sfumato a causa di un consistente numero di casi non rile$v^{2} \mathrm{ti}^{22}$. Con questa precisazione, si possono tuttavia avanzare le seguenti considerazioni. I prefetti dimostrano, in due occasioni su quattro ${ }^{23}$, di avere una minore propensione alla socializzazione in contesti diversi dal ministero di appartenenza. I dirigenti appartenenti ai restanti ministeri condividono invece esperienze di socializzazione anche presso altre strutture.

Passo ora a presentare i dati sulla mobilità dei dirigenti generali (tabb. 6 e 7). Il periodo sul quale sono stati fatti calcoli e valutazioni termina al momento della nomina a prefetto/dirigente generale.

I dati della tabella 6 sono calcolati in base al numero di spostamenti del futuro dirigente generale. Non sono stati presi in considerazione i passaggi dalle strutture centrali a quelle periferiche (e viceversa) dello stesso ministero. L'assunto è che ad una mobilità geografica non corrisponda una mobilità interorganizzativa. Gli spostamenti considerati attengono quindi al passaggio dal ministero di appartenenza (o da altra organizzazione di appartenenza) ad altre strutture della pubblica amministrazione (ministeri, presidenza del consiglio, comitato regionale di controllo, commissariato di governo nella regione), ad enti pubblici, enti territoriali, società del sistema pubblico parallelo, ad altre organizzazioni (organizzazioni di rappresentanza degli interessi, organizzazioni di studio e ricerca).

${ }^{22}$ Il calcolo è stato effettuato sulla percentuale di dati validi.

${ }^{23}$ Vedi la percentuale degli incarichi che i soggetti appartenenti al MI ricoprono nei consigli di amministrazione e negli organi di controllo di organizzazioni diverse da quella di appartenenza. 
TAB. 6. Passaggi di sede

\begin{tabular}{lrcrc}
\hline & MI & MICA & MLPS & Totale \\
\hline nessuno & 11 & 7 & 10 & 28 \\
uno & 8 & 0 & 6 & 14 \\
due & 1 & 0 & 0 & 1 \\
più di cinque & 0 & 0 & 7 & 7 \\
missing & 0 & 3 & 2 & 5 \\
Totale & 20 & 10 & 25 & 55 \\
\hline
\end{tabular}

I dati rivelano che il percorso di carriera di 11 futuri prefetti si dipana interamente nell'ambito del MI. Naturalmente sono frequenti le esperienze presso le prefetture oltre che presso le strutture centrali del ministero ma ciò non sottopone il soggetto ad una diversa socializzazione. Anzi è proprio presso la prefettura che avviene il training iniziale del futuro prefetto. I restanti casi esibiscono una bassissima mobilità.

Il quadro empirico è speculare per ciò che riguarda il MLPS dove un nutrito gruppo di soggetti (sette) totalizza più di cinque spostamenti prima della nomina politica. Le sedi più frequentate sono gabinetti e segreterie dei ministri. Coloro che esibiscono un solo spostamento (sei) hanno la stessa destinazione: la Presidenza del consiglio dei ministri, impegnata attraverso il dipartimento della funzione pubblica, sui temi della contrattazione collettiva nel pubblico impiego. C'è poi un ultimo gruppo di soggetti che dispiega la propria carriera unicamente nell'ambito del ministero. Per quanto riguarda il MICA va detto che le informazioni mancanti riguardano i dirigenti generali provenienti dall'esterno. Gli altri soggetti (sette) mostrano un iter di carriera privo di mobilità.

La tabella 7 riassume i tre profili di carriera emersi dall'analisi.

La prima categoria (interno) è occupata da quei burocrati che hanno compiuto il percorso di carriera esclusivamente nell'ambito del ministero di appartenenza. Presso il MICA e il MI è l'itinerario più frequente. La seconda categoria (interno/esterno) comprende quei soggetti che, attraverso la pratica del fuori ruolo, maturano esperienze presso altre organizzazioni del settore pubblico. Nel caso del MI la destinazione è rappresentata frequentemente - dal Comitato regionale di controllo e dal Commissario di governo presso la regione. Per il MLPS si configurano collocamenti fuori ruolo presso la Presidenza del consi- 
TAB. 7. Percorsi di carriera

\begin{tabular}{lrccc}
\hline & MI & MICA & MLPS & Totale \\
\hline solo interno & 11 & 7 & 10 & 28 \\
interno ed esterno & 9 & 0 & 6 & 15 \\
solo esterno & 0 & 3 & 9 & 12 \\
Totale & 20 & 10 & 25 & 25 \\
\hline
\end{tabular}

glio dei ministri. Il MICA non invia il proprio personale presso altre organizzazioni pubbliche. La terza categoria (esterno) comprende quei profili di carriera che si dispiegano in luoghi diversi dal ministero in cui si va a ricoprire l'incarico di dirigente generale. Questo dato esprime una debolezza della burocrazia nel controllo delle nomine e dunque un successo dell'attore politico. $\grave{E}$ spesso indicativa di un fenomeno di mero transito finalizzato alla acquisizione della qualifica di dirigente generale. Oppure il significato va trovato nel quadro di un legame di fedeltà personale al vertice politico tale per cui alla sostituzione del ministro segue quella del dirigente generale. Quest'ultimo percorso caratterizza la carriera dei dirigenti generali del MLPS $\mathrm{e}$, in misura inferiore, quella dell'alta dirigenza del MICA.

L'esame dello spettro delle attività è stato svolto al fine di accertare il grado di partecipazione della dirigenza generale al policy-making, indicatore del tipo di funzioni effettivamente svolte nel corso della carriera compiuta all'interno di amministrazioni pubbliche. L'operazione di classificazione, il cui risultato è esposto nella tabella 8 , ha tenuto conto delle mansioni svolte dai soggetti durante tutta la loro carriera ${ }^{24}$. Con il concetto di controllo si intende l'attività di verifica della congruenza fra la norma e un atto, e fra la norma e l'attività di soggetti esterni. Essa è spesso associata alla collocazione in line del soggetto. Il concetto di studio e formulazione di politiche, rinvia alle seguenti attività: predisposizione di provvedimenti e disegni di legge, consulenza sulle opzioni di policy, azione come presidenti, segretari, rappresentanti di comitati e commissioni ministe-

${ }^{24} \grave{E}$ stata effettuata una trascrizione non codificata anche del periodo e ambito organizzativo nei quali le attività sono state espletate. Successivamente, il materiale empirico raccolto è stato classificato così come la tabella 8 mostra. In fase di codifica, la lista delle categorie contava anche «controllo+negoziazione» e «negoziazione» che però sono risultate vuote. 
ТАВ. 8. Le attività per ministero

\begin{tabular}{lcccc}
\hline & MI & MICA & MLPS & Totale \\
\hline controllo & 7 & 0 & 1 & 8 \\
controllo+policy & 4 & 2 & 9 & 15 \\
controllo+policy+negoz. & 8 & 3 & 1 & 12 \\
policy & 1 & 1 & 9 & 11 \\
policy+negoziazione & 0 & 4 & 5 & 9 \\
Totale & 20 & 10 & 25 & 55 \\
\hline
\end{tabular}

riali ed interministeriali. La sfera di attività è chiara, ma non trova una rispondenza univoca nella dislocazione strutturale. Il soggetto può occupare, indifferentemente, posizioni di staff o line. Il carattere dell'intervento può essere auto e/o etero-regolativo. Infine, il concetto di negoziazione denota la raccolta di istanze provenienti dagli attori presenti nell'ambiente pertinente del ministero e la risoluzione di vertenze fra parti sociali. Anche in questo caso, il dirigente può essere inserito in staff o line. Infine, si ipotizza che l'attività di negoziazione sia indicativa di un maggior grado di politicità rispetto all'attività di studio e formulazione di policy, a sua volta indicativa di un maggior grado di politicità rispetto all'attività di controllo ${ }^{25}$.

I risultati attinenti alle attività in cui è impegnata la dirigenza generale rivelano una duplice tendenza. In primo luogo emerge un maggior grado - proporzionalmente - di partecipazione all'attività di decision-making presso il MLPS e il MICA, contesti caratterizzati da una forma di specializzazione in merito ad attività di costruzione di programmi e di mediazione fra le parti sociali. In secondo luogo, i vari contesti esibiscono profili differenziati: presso il MI domina un profilo di carattere generalista che denuncia una professionalità costruita nel tempo, attraverso il preliminare impegno su funzioni di controllo; presso il MLPS e, in misura più ridotta, presso il MICA si afferma un profilo specialistico notevolmente influenzato dalle competenze assegnate ai ministeri socio-economici. La natura delle competenze assegnate ai ministeri certamente influenza il grado di partecipazione al decision-making, ma un ruolo considerevole è svolto dagli

\footnotetext{
${ }^{25}$ In definitiva, si ipotizza che la variabile «tipo di funzioni» sia categoriale e ordinata.
} 
TAB. 9. L'estrazione organizzativa per il tipo di attività

\begin{tabular}{lccccc}
\hline & \multicolumn{4}{c}{ Provenienza } & \\
\cline { 2 - 4 } & interna & PA-ord. & PA-par. & altro & Totale \\
\hline controllo & 8 & 0 & 0 & 0 & 8 \\
controllo+policy & 13 & 2 & 0 & 0 & 15 \\
controllo+policy+negoz. & 12 & 0 & 0 & 0 & 12 \\
policy & 5 & 4 & 2 & 0 & 11 \\
policy+negoziazione & 2 & 0 & 4 & 3 & 9 \\
Totale & 40 & 6 & 6 & 3 & 55 \\
\hline
\end{tabular}

Legenda:

$P A$-ord. sta per «altra pubblica amministrazione ordinaria rispetto al ministero di appartenenza»;

$P A-p a r$. sta per «organizzazione appartenente al sistema pubblico parallelo»; altro sta per «altre organizzazioni rispetto a quelle indicate».

incarichi ricoperti nelle strutture di staff e di ausilio degli organi politici del ministero (gabinetto e segreteria). A questo proposito è utile considerare anche la provenienza organizzativa (tab. 9).

Sono pochi (sette) i dirigenti «di carriera» impegnati esclusivamente in funzioni di policy-making e di negoziazione. L'associazione con l'estrazione organizzativa è tale da escludere una forma di socializzazione inside dettata unicamente dalle competenze funzionali del contesto ministeriale. Al contrario, essa mostra che tali attività sono concentrate presso coloro che provengono dall'esterno dell'organizzazione di appartenenza e che prima di acquisire la qualifica di dirigente generale hanno occupato posizioni vicine al vertice politico.

Un dato da sottolineare, in ogni caso, è la natura composita dell'attività di coloro che hanno attraversato tutte le qualifiche della carriera e, dall'altro lato, la natura esclusiva dell'attività riscontrabile in coloro che provengono da organizzazioni diverse dal ministero in cui sono attualmente incardinati.

Per evidenziare qualche altro tratto dell'identikit professionale è utile associare il tipo di laurea al grado di partecipazione al policy-making (vedi tab. 10). Innanzitutto però la distribuzione monovariata mette in luce un ridimensionamento, rispetto ai dati solitamente presentati dalla letteratura, del peso della laurea in legge, tanto più significativo in quanto i dati della dirigenza del MICA e del MLPS esauriscono l'intera popolazione. In 
TAB. 10. Tipo di laurea per ministero

\begin{tabular}{lrrrr}
\hline & Totale & MI & MICA & MLPS \\
\hline giurisprudenza & 34 & 19 & 3 & 12 \\
scienze politiche & 4 & 1 & 1 & 2 \\
economia e statistica & 14 & 0 & 3 & 11 \\
ingegneria & 3 & 0 & 3 & 0 \\
Totale & 55 & 20 & 10 & 25 \\
\hline
\end{tabular}

TAB. 11. Tipo di laurea per tipo di attività

\begin{tabular}{lcccc}
\hline & giuridica & non-giuridica & Totale & $\%$ \\
\hline controllo & 8 & 0 & 8 & 14,5 \\
controllo+policy & 10 & 5 & 15 & 27,3 \\
controllo+policy+negoz. & 9 & 3 & 12 & 21,8 \\
policy & 3 & 8 & 11 & 20,0 \\
policy+negoziazione & 4 & 5 & 9 & 16,4 \\
Totale & 34 & 21 & 55 & 100,0 \\
\hline
\end{tabular}

secondo luogo, la tabulazione incrociata mette in rilievo, senza sorpresa, che tutti i prefetti - tranne uno - sono dotati di laurea in giurisprudenza. L'interesse va rivolto però ai valori espressi dal MICA e dal MLPS, perché indicativi della rottura del monopolio della conoscenza giuridica in ambito ministeriale.

In entrambi i ministeri socio-economici la maggioranza dei dirigenti generali possiede una laurea non giuridica indicativa di una formazione necessaria a comprendere ed elaborare i contenuti tecnico-specialistici delle rispettive aree di intervento.

L'interpretazione sembra confermata dall'associazione riscontrabile nella tabella 11 fra partecipazione all'attività di policy e negoziazione e una formazione diversa da quella giuridica.

I dati mostrano una tendenza accentuata alla sostituzione della conoscenza giuridica man mano che si passa dalle attività di controllo alle attività di policy e negoziazione. È questo un interessante indizio di coerenza fra tipo di compito assegnato all'istituzione e tipo di competenza disponibile all'interno dell'organizzazione. Va infatti detto che le due coerenze possibili si stabiliscono, rispettivamente, fra un compito di carattere amministrativo ed una competenza di tipo generalista e fra un 
compito di carattere tecnico-operativo ed una competenza specialistica. Va però aggiunto che $\mathrm{i}$ due ministeri socio-economici sono ricorsi - anche se in misura diversa perché più massiccia al MLPS - a competenze maturate all'esterno dell'istituzione. E questo evidentemente testimonia la carenza di professionalità tecnico-specialistiche prodotte in via diretta dall'istituzione.

Consideriamo ora l'età e la sua relazione con il grado di partecipazione ai processi decisionali (tabb. 12 e 13).

Un risultato appare interessante: la presenza di 12 dirigenti generali - il 21,8\% - con un'età inferiore ai 50 anni. Questo dato, anche se interpretato alla luce delle novità apportate dal DPR 748/1972 sulla dirigenza, che ha ampliato l'organico prevedendo oltre all'incarico di direttore generale quello di consigliere ministeriale, rappresenta in ogni caso un mutamento di rotta rispetto alla tendenza in vigore fino alla fine degli anni '70. In quest'ultimo caso, la nomina raggiungeva il soggetto nel suo ultimo decennio di attività prima della pensione ${ }^{26}$. In secondo

TАв. 12. L'età per ministero

\begin{tabular}{lcccc}
\hline & MI & MICA & MLPS & Totale \\
\hline fino a 44 anni & 0 & 1 & 4 & 5 \\
da 45 a 49 anni & 1 & 2 & 4 & 7 \\
da 50 a 54 anni & 8 & 1 & 6 & 15 \\
da 55 a 59 anni & 9 & 4 & 7 & 20 \\
60 anni e oltre & 2 & 2 & 4 & 8 \\
Totale & 20 & 10 & 25 & 55 \\
\hline
\end{tabular}

TAB. 13. L'età per tipo di attività

\begin{tabular}{lcccccc}
\hline & fino 44 anni & $45 / 49$ & $50 / 54$ & $55 / 59$ & $60 \mathrm{e}+$ & Totale \\
\hline controllo & 0 & 0 & 5 & 2 & 0 & 8 \\
controllo+policy & 0 & 3 & 3 & 6 & 3 & 15 \\
contr.+policy+negoz. & 0 & 0 & 2 & 7 & 3 & 12 \\
policy & 3 & 2 & 4 & 0 & 2 & 11 \\
policy+negoziazione & 2 & 2 & 0 & 5 & 0 & 9 \\
Totale & 5 & 7 & 15 & 20 & 8 & 55 \\
\hline
\end{tabular}

${ }^{26}$ Il primo riferimento è a Cassese $(1981,228)$. I dati, al 1962, relativi ad un campione di 150 direttori generali rivelano che il $90 \%$ ha più di 50 anni e circa il $55 \%$ è nel decennio precedente all'età pensionabile. 
luogo, va annotato che otto dei dodici dirigenti con età inferiore ai 50 anni appartengono al Ministero del lavoro, tre al Ministero dell'industria e uno al Ministero dell'interno.

Passo ora a commentare l'incrocio con il tipo di attività (tab. 13). Sappiamo che non ci sono prefetti con meno di 45 anni e che la grande maggioranza (17 su 20$)$ ha una età compresa fra i 50 e i 60 anni. L'assunzione di funzioni prefettizie presso una sede provinciale - a cui sono spesso associati interventi di controllo, elaborazione di decisioni e mediazione con gli attori locali - avviene, generalmente, nella fase terminale della carriera (dai 55 ai 65 anni). Questo spiega l'affollamento delle fasce corrispondenti ad una età superiore a 50 anni. Per ciò che riguarda gli altri due ministeri si può facilmente ipotizzare una relazione significativa fra la giovane età (sotto i 50 anni) di nove dirigenti e il loro impegno in attività di elaborazione di policy e di negoziazione con le parti sociali. Ciò conferma peraltro le caratteristiche di un ingresso nella dirigenza generale che prescinde dalla anzianità di servizio così come da altre caratteristiche di un profilo «amministrativo».

Concludo la ricostruzione del profilo professionale del dirigente con alcune considerazioni sui settori di attività (tab. 14) presso i quali ognuno ha maturato la propria esperienza.

Innanzitutto va sottolineata la differenziazione fra MI e MICA da un lato e MLPS dall'altro lato. Tutti i dirigenti generali - tranne 2 - dei primi due ministeri sono accomunati da esperienze professionali maturate nell'ambito dei settori operativi. Per contro, né presso il MI, né presso il MICA, le strutture di supporto costituiscono il settore di addestramento all'alta dirigenza. Al contrario, presso il MLPS, il $40 \%$ della dirigenza generale ha frequentato esclusivamente strutture di staff, presso le quali, evidentemente, la socializzazione intraorganizzativa ha minor impatto data la predominanza di logiche politiche. Necessaria, nel

TAB. 14. I settori ricoperti dall'alta dirigenza per ministero

\begin{tabular}{lcccrrrrr}
\hline & MI & $\%$ & MICA & $\%$ & MLPS & $\%$ & Totale & $\%$ \\
\hline line & 9 & 45,0 & 6 & 60,0 & 0 & 0,0 & 15 & 27,3 \\
staff & 2 & 10,0 & 0 & 0,0 & 10 & 40,0 & 12 & 21,8 \\
esperienza mista & 9 & 45,0 & 4 & 40,0 & 15 & 60,0 & 28 & 50,9 \\
Totale & 20 & & 10 & & 25 & & 55 & 100,0 \\
\hline
\end{tabular}


caso del MICA, è l'esperienza all'interno dei settori operativi connotata dal dialogo incessante fra ministero e attori socio-economici.

Termino con due considerazioni di sintesi: la prima dedicata al profilo del dirigente generale, la seconda riferita all'analisi dei dati fin qui emersi.

Sul primo aspetto, i prefetti rivelano un profilo generalista che prevede una politicità del ruolo solo nella fase terminale della carriera per «stratificazione» delle esperienze maturate nel corso di un lungo iter il quale si dipana attraverso incarichi per la gran parte di carattere operativo. Circa un terzo dei dirigenti del MLPS manifesta invece una professionalità politica, costruita in un arco di tempo piuttosto breve (sono tutti piuttosto giovani) nell'ambito di strutture di staff di ministri e sottosegretari. Il nucleo «interno» della dirigenza del MLPS si differenzia da questo profilo per l'età più matura, la laurea giuridica e un minore impegno nell'attività di policy e negoziazione. Il profilo dei dirigenti del MICA è a sua volta caratterizzato da una professionalità tecnica: sono stati impegnati in strutture operative anche se non nell'ambito del ministero in cui risultano incardinati e la loro partecipazione all'attività decisionale non si esplica nell'ambito degli organi politici bensì in quello dei ruoli previsti dalla line.

Dal quadro d'insieme sembra emergere una maggiore tenuta del modello burocratico di reclutamento, socializzazione e carriera rispettivamente presso - in scala decrescente - il Ministero dell'interno, quindi il Ministero dell'industria e, infine, il Ministero del lavoro. L'impressione trova una conferma se $i$ dati vengono interpretati alla luce dei due modelli denominati inside e outside (fig. 1). La figura consente di richiamare l'atten-

\begin{tabular}{|c|c|c|c|}
\hline & MI & MICA & MLPS \\
\hline provenienza & inside & outside & outside \\
\hline incarico politico & inside & inside & outside \\
\hline accesso alla dirigenza & inside & outside & outside \\
\hline incarichi & inside & outside & outside \\
\hline mobilità & inside & intermedio & outside \\
\hline attività & inside & intermedio & outside \\
\hline laurea & inside & outside & outside \\
\hline età & inside & intermedio & outside \\
\hline settore & inside & inside & outside \\
\hline
\end{tabular}

FIG. 1. Modelli di reclutamento e carriera. 
zione su due importanti aspetti della ricerca empirica fin qui condotta. In primo luogo la «polarizzazione» fra Ministero dell'interno e Ministero del lavoro. Il MI appare dotato di un sistema che forgia il proprio personale. Al contrario, il MLPS è un ambiente aperto ad ospitare personale proveniente da organizzazioni esterne. In secondo luogo va riservata attenzione alla posizione intermedia del Ministero dell'industria che, pur essendo assimilabile al Ministero del lavoro per ciò che concerne il dettato normativo sull'inquadramento del personale dirigenziale e i criteri che ne regolano reclutamento e avanzamento, dimostra però una parziale tenuta del modello burocratico.

\section{La forza dell'istituzione}

Siamo partiti dal presupposto che l'organizzazione normalmente operi sul proprio personale attraverso una tripla modali$\operatorname{tă}^{27}:$ a) un sistema di selezione e reclutamento volto ad escludere il personale incongruo ovvero privo dei requisiti formali/informali di ingresso; $b$ ) una socializzazione volta all'apprendimento di valori, consuetudini, prassi istituzionali, tale da sviluppare un sentimento di appartenenza istituzionale; $c$ ) la trasmissione, sostenuta da un sistema di premi-punizioni, di regole che permettono di discriminare fra comportamenti ammissibili e comportamenti inammissibili.

Sotto questo profilo, le patologie delle relazioni fra istituzioni e personale sono da ricondurre, da un lato, alla debolezza istituzionale, dall'altro, all'incompatibilità fra caratteristiche del personale e valori fondanti l'istituzione. Dalla prima circostanza deriva l'incapacità, da parte dell'istituzione, di socializzare il personale che la incarna; la seconda circostanza si presenta qualora un'istituzione ospiti al proprio interno, personale marcato $\mathrm{da}$ eterogeneità rispetto ai valori che legittimano l'istituzione e le sue modalità operative ${ }^{28}$. 280)

${ }^{27}$ Le considerazioni sul rapporto istituzione-personale sono riprese da Cotta (1979,

${ }^{28} \mathrm{Ci}$ si riferisce al mancato sviluppo di diffuse lealtà nei confronti di un insieme di valori propri dell'istituzione. A tal proposito, ridefinendo ed adattando il pensiero di Blau (1964), Freddi specifica che «l'ideologia organizzativa - una combinazione, o un complesso, di norme e orientamenti interiorizzati dai partecipanti organizzativi - viene sostenuta da due tipi principali di valori: i valori legittimanti, ed i valori che giustificano i criteri adottati per la differenziazione e la stratificazione dei partecipanti» $(1977,25)$. 
Tali considerazioni si ispirano ai contributi in tema di istituzionalizzazione ${ }^{29}$ che hanno avuto il merito di concentrare l'attenzione sia sulle caratteristiche proprie del rapporto fra organizzazione e ambiente, sia sulle caratteristiche interne al sistema.

Il concetto qui prescelto ai fini dell'analisi del rapporto fra personale e istituzione è quello di forza dell'organizzazione quale capacità di controllo di alcune dimensioni cruciali dell'assetto organizzativo. L'ipotesi è che a tale capacità corrisponda la compattezza dell'istituzione ${ }^{30}$. Quest'ultimo concetto intende fare riferimento più che ad una capacità di adattamento esterno dell'organizzazione, alla sua capacità di integrazione interna. I due aspetti risultano necessariamente connessi, sia sul piano analitico sia sul piano empirico ${ }^{31} \mathrm{ma}$, ai fini della presente analisi, l'ambito di ricerca è stato circoscritto alla dimensione interna della compattezza dell'istituzione.

Il concetto di compattezza individua allora una serie di aspetti:

- la capacità dell'organizzazione di agire come un sistema

${ }^{29}$ Vedi per tutti Huntington per il quale «l'istituzionalizzazione è il processo tramite il quale organizzazioni e procedure acquistano validità e stabilità» $(1968,25)$. La trattazione del concetto di istituzionalizzazione proposta dall'autore individua quattro dimensioni: la flessibilità o adattamento all'ambiente, la complessità strutturale, l'autonomia nei confronti di altre organizzazioni e procedure, la compattezza o coerenza interna. L'assunto teorico sottostante è che valori elevati sulle quattro dimensioni indichino un elevato livello di istituzionalizzazione della organizzazione.

${ }^{30}$ Per il concetto di compattezza mi sono ispirata ad Huntington, per il quale «più un'organizzazione è unita e compatta e più il suo livello di istituzionalizzazione è elevato; maggiore è invece la sua frammentazione e minore è il suo grado di istituzionalizzazione. (...) un'organizzazione efficiente richiede un consenso sostanziale sui confini funzionali del gruppo e sulle procedure dirette a risolvere le controversie all'interno di questi confini. (...) In teoria un'organizzazione può essere autonoma senza essere compatta e compatta senza essere autonoma. Tuttavia nella realtà queste due caratteristiche sono spesso collegate tra di loro in modo molto stretto. L'autonomia diventa uno strumento di compattezza, in quanto rende l'organizzazione capace di sviluppare uno spirito e uno stile che diventano caratteri distintivi del suo comportamento» $(1968,34-35)$.

${ }^{31} \mathrm{Su}$ questo punto vedi Panebianco per il quale «l'istituzionalizzazione organizzativa, nell'accezione qui accolta può essere misurata, essenzialmente, lungo due dimensioni: 1) il grado di autonomia dall'ambiente che l'organizzazione ha sviluppato; 2) il grado di sistemicità, di interdipendenza fra le diverse parti dell'organizzazione. La dimensione autonomia/dipendenza si riferisce alla relazione che l'organizzazione instaura con l'ambiente esterno. (...) La seconda dimensione dell'istituzionalizzazione, il grado di sistemicità, si riferisce alla coerenza strutturale interna alla organizzazione. (...) Le due dimensioni della istituzionalizzazione tendono ad essere collegate fra loro nel senso che un basso grado di sistemicità organizzativa implica spesso una debole autonomia dall'ambiente. E viceversa» (Panebianco 1982, 113-114 e 116-117). 
integrato, il che implica una stretta connessione fra le diverse unità del sistema e la presenza di gerarchia;

- la presenza di meccanismi di difesa rispetto all'ingerenza di forze esterne, il che implica la presenza di regole formali/informali in grado di discriminare l'accesso dei candidati all'élite ministeriale;

- la possibilità di disporre stabilmente delle risorse necessarie al funzionamento, il che implica l'assegnazione stabile di un proprio corpo di funzionari e la possibilità di attingere regolarmente informazioni e servizi da propri uffici di staff;

- la presenza di meccanismi di socializzazione capaci di sviluppare uno spirito e uno stile distintivi del comportamento.

Sia la particolarità della socializzazione del personale, sia la presenza di regole a garanzia del reclutamento interno dell'élite ribadiscono i confini organizzativi e rafforzano l'identità e il senso di appartenenza.

La compattezza del ministero è stata valutata facendo riferimento alle dimensioni sopraelencate al fine di produrre un ordine di allineamento delle tre organizzazioni.

Il concetto è stato articolato in vari indicatori, il primo dei quali fa riferimento alla possibilità di disporre di un proprio corpo di funzionari. La seguente tabella (tab. 15) permette di valutare, soprattutto in base al dato percentuale, il peso che altre istituzioni esercitano sui tre ministeri attraverso la quota di coloro che, sottoposti all'istituto del comando ${ }^{32}$ da parte delle organizzazioni di appartenenza, prestano la loro opera nel ministero in esame.

Insieme struttura centrale e struttura periferica del MICA ospitano circa 1500 unità di personale di cui circa 200 esterni provenienti in larga parte dagli enti vigilati. Il personale impiegato al MLPS ammonta a circa 16.000 unità di cui circa 400 appartengono ad altre amministrazioni pubbliche. Indicativo di una tendenza opposta è il dato esibito dall'amministrazione civile del MI che impiega quasi 19.000 unità di cui però solo 85 sono esterne.

Il secondo indicatore descrive lo stato degli uffici di staff. Preoccupanti sono le condizioni in cui versano gli uffici dei due ministeri socio-economici: l'uffico studi del MICA è disattivato e

\footnotetext{
${ }^{32}$ L'istituto del comando si configura quando «l'impiegato è destinato a prestare servizio presso altra amministrazione statale» (Virga 1973, 651).
} 
TAB. 15. Il personale per ministero (1)

\begin{tabular}{lccc}
\hline & impiegato & «comandato» & \% di unità comandate \\
\hline MICA & 1.500 & 200 & 13,3 \\
MLPS & 16.000 & 400 & 2,5 \\
MI (2) & 19.000 & 85 & 0,4 \\
\hline
\end{tabular}

(1) I dati si riferiscono rispettivamente al 1989 per il MICA e il MLPS e al 1991 per il MI.

(2) Il ministero nel suo complesso (amministrazione civile, corpo nazionale dei vigili del fuoco, polizia di stato) conta circa 129.000 unità. Qui interessa solo l'amministrazione civile.

al MLPS sono assenti sia l'ufficio studi che l'ufficio legale. Le direzioni generali del MLPS ${ }^{33}$ anziché essere dotate di vere e proprie strutture di staff ospitano al proprio interno un grande numero di organismi collegiali costituiti, oltre che da funzionari del ministero, da rappresentanti di associazioni di interessi. Quelle del MICA $^{34}$ sono più attrezzate in quanto almeno un ufficio di staff è previsto in ogni direzione generale; tutte le direzioni generali del $\mathrm{MI}^{35}$ sono dotate di varie strutture di staff.

Il terzo indicatore della compattezza dell'organizzazione attiene alla presenza di meccanismi di difesa rispetto all'ingerenza di forze esterne. Né al MICA né al MLPS si rintracciano norme di difesa dell'élite da immissioni esterne. Viceversa, il corpo dei

${ }^{33}$ Le direzioni generali del MLPS sono: la direzione generale degli affari generali e del personale, la direzione generale dei rapporti di lavoro, la direzione generale per l'impiego, la direzione generale della previdenza e assistenza sociale, la direzione generale per la cooperazione, l'ufficio centrale per l'orientamento e la formazione professionale dei lavoratori, la direzione generale dell'osservatorio del mercato del lavoro. Per maggiori dettagli vedi Mariani (1988).

${ }^{34}$ Le direzioni generali del MICA sono: la direzione generale degli affari generali, la direzione generale delle fonti di energia e industria di base, la direzione generale della produzione industriale, la direzione generale delle miniere, la direzione generale del commercio interno e del consumo industriale, la direzione generale delle assicurazioni private e di interesse collettivo, l'ufficio centrale brevetti. Per maggiori dettagli vedi Sacco e Sensale (1986).

${ }^{35} \mathrm{Ci}$ si riferisce alle strutture di staff interne al dipartimento di pubblica sicurezza, alla direzione generale per l'amministrazione generale e per gli affari del personale, alla direzione generale dell'amministrazione civile, alla direzione generale degli affari dei culti, alla direzione generale dei servizi civili, alla direzione generale della protezione civile e dei servizi anti incendi. I restanti due uffici centrali del $\mathrm{MI}$ - l'ufficio centrale per gli affari legislativi e le relazioni internazionali e l'ufficio centrale per i problemi delle zone di confine e delle minoranze etniche - sono essi stessi uffici di staff. Per le informazioni sulla struttura organizzativa del Ministero dell'interno vedi Meoli (1986). 
prefetti ha ottenuto nel 1937 una norma, confermata con il testo unico del 1957, che impedisce la nomina di più dei $2 / 5$ dei prefetti fra il personale non di carriera. La prassi vigente presso il ministero è quella di calcolare la quota da destinare ad immissioni di soggetti esterni sulla base dei posti vacanti e non sull'organico, ottenendo in tal modo una vistosa riduzione delle possibilità di reclutamento di personale esterno.

Relativamente alla presenza di gerarchia, va sottolineato che il ministero generalmente si articola in direzioni generali, divisioni e sezioni. Allo svolgimento delle attività lungo tale linea operativa presiede il criterio dell'interdipendenza seriale ${ }^{36}$, tale per cui la prestazione $B$ può essere espletata solo successivamente alla completa riuscita della prestazione $A$, che a sua volta dipende da una precedente prestazione e così di seguito.

Le informazioni relative all'articolazione gerarchica dei vari ministeri permettono dunque di valutare il maggiore o minore orientamento di ciascuna organizzazione verso la gerarchia. L'ipotesi è che l'interdipendenza fra le varie unità sia tanto più stringente quanto più numerosi sono i livelli gerarchici presenti.

Sotto questo profilo il MI mostra una atipicità: la presenza di due livelli in più - la direzione centrale e il servizio - che alterano la struttura-tipo del ministero, articolata normalmente su direzione generale, divisione e sezione, e che rafforzano la gerarchia. Al contrario, sia presso il MICA che presso il MLPS esistono tre livelli gerarchici (non cinque come al MI), in aderenza alla struttura-tipo del ministero. Tale peculiarità del MI denota una interdipendenza fra unità organizzative poste lungo la linea operativa più stringente rispetto a quella dei restanti ministeri.

Concludo con il riscontro della presenza di particolari meccanismi di socializzazione capaci di sviluppare uno spirito e uno stile distintivi del comportamento rafforzando l'identità e il senso di appartenenza organizzativa. A questo proposito va detto che il MI oltre ad aver conservato una carriera prefettizia con criteri di promozione e stato giuridico particolari, prevede un periodo di training in prefettura per tutti coloro che accedono alla prima qualifica della carriera prefettizia orientato a stimolare una formazione generalista, dato il ruolo e le funzioni del mi-

${ }^{36}$ Il richiamo è all'associazione di Thompson (1967) fra tipi di tecnologia, quale modalità di trasformazione di input in output (di concatenamento, di mediazione, intensiva) e tipi di interdipendenza fra soggetti (seriale, standardizzata, combinatoria di una pluralità di tecniche sulla base del feed-back). 


\begin{tabular}{llcc}
\hline & \multicolumn{3}{c}{ Controllo delle fonti di incertezza } \\
\cline { 2 - 4 } & alto & intermedio & basso \\
\hline - personale & MI & MLPS & MICA \\
- uffici & MI & MICA & MLPS \\
- difesa dei confini & MI & & MICA-MLPS \\
- socializzazione & MI & & MICA-MLPS \\
- gerarchia & MI & & MICA-MLPS \\
\hline
\end{tabular}

FIG. 2. Forza dell'istituzione.

nistero. In secondo luogo il ministero, fin dal 1980, dispone di una struttura di formazione interna - la Scuola superiore del Ministero dell'interno - che provvede alla formazione e all'aggiornamento professionale del personale dell'amministrazione. Meccanismi e strutture di socializzazione speciali non sono rintracciabili presso i restanti due ministeri.

La figura 2 permette di riepilogare i risultati dell'analisi della forza espressa dai tre ministeri.

Tutte le dimensioni considerate mostrano la regolare collocazione del MI in posizione di maggior grado di compattezza rispetto agli altri due ministeri. Ospita un basso numero di personale comandato; tutti gli uffici previsti da fonti normative e da regolamenti interni sono attivati; il ministero addestra direttamente il proprio personale attraverso la Scuola superiore del Ministero dell'interno; ha una carriera prefettizia con criteri di promozione e stato giuridico particolari e regole di ferrea limitazione di immissioni esterne nell'élite. Il MICA e il MLPS sono, al contrario, accomunati da rilevante quota di personale comandato da altre amministrazioni; scontano la disattivazione di uffici di raccolta e trattamento di informazioni e attingono per questo tipo di competenze a soggetti estranei al ministero. Inoltre, dall'analisi dei meccanismi di socializzazione dei due ministeri non emerge la presenza di prassi consuete e reiterate nel tempo, né quella di norme di difesa della leadership ad estrazione interna.

\section{Burocrazia e politica: un confronto tra $i$ casi}

Il punto di avvio di questa ricerca è stata l'ipotesi che la burocrazia ministeriale non fosse un'entità indifferenziata ma, al contrario, presentasse al proprio interno linee di differenziazione lungo le dimensioni del reclutamento, della socializzazione e della carriera. 
Si è partiti dall'esame delle proprietà dell'élite al fine di accertare gli effetti prodotti da una duplice modalità di governo del personale sulla compattezza dell'istituzione.

L'ipotesi che ha guidato la ricerca è che tanto più il reclutamento, la socializzazione e la carriera sono controllati dall'istituzione amministrativa, tanto più il personale risulta influenzato $\mathrm{da}$ logiche di appartenenza istituzionale, tanto più forte si rivela l'istituzione. Viceversa, tanto meno il reclutamento, la socializzazione e la carriera sono controllati, tanto più il personale risulta esposto a logiche di comportamento estranee al contesto istituzionale di appartenenza, tanto più l'istituzione si dimostra debole in quanto perde il controllo di importanti risorse organizzative.

La ricognizione delle conseguenze che i diversi meccanismi organizzativi hanno sulla compattezza e unità delle tre istituzioni ha riproposto la differenziazione fra il Ministero dell'interno da un lato, e gli altri due ministeri, dall'altro lato, dando forza all'ipotesi iniziale che le modalità di governo del personale incidano sul contesto istituzionale.

Va allora sottolineato da un lato il legame fra i meccanismi di governo del personale del Ministero dell'interno e la compattezza dell' 'istituzione e, dall'altro lato, il legame fra il modello di reclutamento e carriera del personale dei due ministeri socioeconomici e la debolezza dell' istituzione.

Il primo ministero guida i processi di socializzazione del personale nel richiamo continuo alle doti e alle qualità del prefetto in sede: lealtà nei confronti del governo e autonomia da esercitare nel rispetto dei valori tradizionali dell'istituzione.

Negli altri due ministeri il personale esibisce caratteristiche di debole socializzazione organizzativa in un contesto istituzionale meno forte rispetto a quello del MI, incerto, poco controllato dalla burocrazia interna. Il grado di compattezza che MICA e MLPS hanno sviluppato è piuttosto basso. Le risorse (umane e materiali) non sono integralmente prodotte all'interno ma vengono in buona parte attinte presso il contesto esterno, dimostrando una situazione di dipendenza da variabili che si sottraggono al controllo dell'istituzione amministrativa.

È possibile a questo punto specificare una delle ipotesi che, presente da tempo nella letteratura, ha subìto però pochi controlli: la configurazione delle proprietà organizzative rivela le strategie di controllo sulla burocrazia da parte di attori non-burocratici. Criteri formali e procedurali di reclutamento e avan- 
zamento non necessariamente sono rivolti a conseguire l'efficienza dell'istituzione, ma piuttosto a conseguire l'autonomia dalla politica ${ }^{37}$.

Sul versante delle strategie di controllo dell'élite burocratica da parte di attori non-burocratici è evidente che il Ministero dell'interno - a paragone degli altri due - esibisce caratteristiche più accentuatamente simili a quelle proprie del modello originario dell'apparato amministrativo. Su questi requisiti quindi sembra poggiare la strategia di controllo basata sull'auto-regolazione burocratica. La sfera di proposta e selezione dei candidati alla nomina di prefetto è controllata dalla burocrazia del ministero anche se, da alcune interviste realizzate con testimoni privilegiati del MI, emerge una inversione di tendenza che, da una decina di anni a questa parte, vede crescere le interferenze del vertice politico $^{38}$. Per contro, il Ministero dell'industria e il Ministero del lavoro evidenziano una disarticolazione del modello legale-razionale della burocrazia, che è probabile abbia attivato uno degli effetti constatati da questa ricerca: tanto più l'organizzazione burocratica - in questo caso i due ministeri socio-economici - risulta marcata da automatismo nei sistemi di ingresso e progressione, tanto più consente l'intervento degli organi politici (ministro, gabinetto del ministro, segreterie particolari di ministro e sottosegretari). Non vi è dubbio che, almeno in parte, soprattutto presso il MLPS, la strategia di controllo della burocrazia risponda ad una modalità di patronage. Il reclutamento su basi politiche è d'altra parte reso agevole da una debole compattezza dell'istituzione nel cui ambito la burocrazia interna è costretta ad accettare nomine che rispondono sostanzialmente a criteri politici imposti da organismi esterni (partiti, sindacati).

Sia il MICA, sia il MLPS ospitano, peraltro, soggetti che simultaneamente ricoprono cariche presso il ministero e presso organizzazioni socio-economiche che fanno parte dell'ambiente al quale il ministero rivolge la propria azione. Si tratta, in parte di soggetti la cui migrazione dagli enti pubblici economici si è ri-

\footnotetext{
${ }^{37}$ Mi riferisco ad una delle ipotesi avanzate da Bacharach e French (1981) secondo la quale $\mathrm{i}$ criteri formali di reclutamento non garantiscono la capacità bensì la neutralità.

${ }^{38}$ Questo vale anche per il ruolo di capo di gabinetto che in termini di status è all'incirca pari ordinato al ruolo di capo di polizia-direttore generale del Dipartimento di pubblica sicurezza del MI. Da sempre controllato dalla burocrazia interna, comincia ad ospitare soggetti esterni che non sono consiglieri di Stato. In ogni caso, l'alto numero di posti di prefetto rende l'arena meno conflittuale e più pronta ad ospitare un gioco a somma positiva.
} 
solta nel conseguimento di cariche operative all'interno del MICA, ed in parte sono individui che, provenendo dalle associazioni di rappresentanza degli interessi, sono approdati ad incarichi di studio e ricerca presso il MLPS. La letteratura sulle «figure di confine ${ }^{39}$ insegna che la conoscenza delle esigenze degli attori socio-economici, unitamente alla responsabilità diretta nell'ambito del ministero, mette tali soggetti in grado di ridurre l'incertezza e l'ambiguità delle percezioni reciproche agevolando l'interazione e le eventuali transazioni. Al fine di valutare se da tale prassi scaturiscano effetti positivi o, al contrario, negativi in termini di rendimento istituzionale, occorrerebbe però un supplemento di indagine volto ad accertare la natura della transazione fra istituzione e soggetti che di provvedimenti attuativi necessitano per svolgere le loro attività.

\section{Riferimenti bibliografici}

Aberbach, J.D., R.D. Putnam e B.A. Rockman (1981), Bureaucrats and Politicians in Western Democracies, Cambridge, Harvard University Press.

Aldrich, H. (1979), Organizations and Environments, Englewood Cliff, N.J., Prentice Hall.

Ammassari, P., F. Garzonio e F. Ferraresi (1969), Il burocrate di fronte alla burocrazia, Archivio ISAP, Milano, Giuffrè.

Bacharach, S.B. e J.L. French (1981), Role-allocation process in public bureaucracies, in «Administration and Society», 4, pp. 399-426.

Bagnasco, A. (1977), Tre italie: la problematica territoriale dello sviluppo italiano, Bologna, Il Mulino.

Blau, P. (1964), Exchange and Power in Social Life, New York, Wiley.

Cassese, S. (1977), Questione amministrativa e questione meridionale. Dimensioni e reclutamento della burocrazia dall'unità a oggi, Milano, Giuffrè.

${ }^{39} \mathrm{Ci}$ si riferisce alla letteratura sui boundary roles. Il concetto è stato formulato da Evan (1967) e serve ad individuare coloro che ricoprono i ruoli di confine dell'organizzazione. La scoperta che alcuni soggetti fossero elementi di mediazione nei rapporti interorganizzativi ha comportato un approfondimento da parte di Aldrich (1979) che, nell'ambito del modello di dipendenza dalle risorse, ha intravisto il ruolo strategico dei boundary roles nella capacità di acquisire presso l'ambiente risorse scarse necessarie alla sopravvivenza dell'organizzazione. Tali individui assumono un ruolo di snodo negli scambi inerenti il flusso delle risorse salienti per le organizzazioni. Anche Crozier (1978), dopo aver introdotto il concetto di ambiente pertinente inclusivo di attori sociali i cui comportamenti influenzano la capacità dell'organizzazione di realizzare le proprie performances, fa riferimento alla stabilizzazione dei rapporti di potere fra organizzazione e ambiente pertinente ricorrendo al concetto di relé. 
- (1981), Grandezza e miserie dell'alta burocrazia italiana, in «Politica del diritto», 2-3, pp. 219-261.

- (1983), Il sistema amministrativo italiano, Bologna, Il Mulino.

- (1985), La formazione dei funzionari amministrativi. Un confronto internazionale, in «Politica del diritto», XVI, 4, pp. 677-689.

Casula, P. (1972), I prefetti nell'ordinamento italiano. Aspetti storici e tipologici, Milano, Giuffrè.

Cerase, F.P. (1990), Un'amministrazione bloccata: pubblica amministrazione e società nell'Italia di oggi, Milano, F. Angeli.

Cotta, M. (1979), Classe politica e parlamento in Italia, 1946-1976, Bologna, Il Mulino.

Crozier, M. (1963), Le phénomène bureaucratique, Paris, Seuil; trad. it. Il fenomeno burocratico, Milano, Etas Kompass, 1969.

Crozier, M. e E. Friedberg (1977), L'acteur et le systeme. Les contraintes de l'action collective, Paris, Seuil; trad. it. Attore sociale e sistema, Milano, Etas Libri, 1978.

Emerson, R.M. (1962), Power dependence relations, in «American Sociological Review», 27, 1962, pp. 31-40.

Etzioni, A. (1964), Modern Organizations, New Jersey, Prentice-Hall, Inc., Englewood Cliffs; trad. it. Sociologia dell'organizzazione, Bologna, Il Mulino, 1967.

Evan, W. (1967), The Organization-Set, in J.D. Thompson (a cura di), Approaches to Organizational Design, Pittsburgh, University of Pittsburgh Press, pp. 173-191.

Ferraresi, F. (1980), Burocrazia e politica in Italia, Bologna, Il Mulino.

Freddi, G. (1977), Tensioni e conflitto nella magistratura, Bari, Laterza.

- (1968), L'analisi comparata dei sistemi burocratici pubblici, Milano, Giuffrè.

- (1982), Vincoli storico-strutturali sulla prestazione delle burocrazie legali-razionali, in «Rivista Italiana di Scienza Politica», 2, pp. 183-211.

- (1986), Governabilità democratica e razionalità amministrativa, in «Rivista Trimestrale di Scienza dell'Amministrazione», 1, pp. 754.

- (1989), Burocrazia, democrazia e governabilità, in G. Freddi (a cura di), Scienza dell'amministrazione e politiche pubbliche, Roma, Nuova Italia Scientifica, pp. 19-65.

Gouldner, A. (1957), Cosmopolitans and Locals: Toward an Analysis of Latent Social Roles, in «Administrative Science Quarterly», 2.

Guarnieri, C. (1977), La politicizzazione della burocrazia pubblica, in «Rivista Trimestrale di Scienza dell'Amministrazione», 3, pp. 435663.

- (1988), Burocrazie pubbliche e consolidamento democratico: il caso italiano, in «Rivista Italiana di Scienza Politica», 1, pp. 73-103. 
Huntington, S.P. (1968), Political Order in Changing Societies, New Haven, Yale University Press; trad. it. Ordinamento politico e mutamento sociale, Milano, F. Angeli, 1975.

Mariani, F. (1988), L'organizzazione centrale e periferica del ministero del lavoro e della previdenza sociale, Napoli, Simone.

Mayntz, R. (1978), Soziologie der offlentlichen verwaltung, Heidelberg, C.F. Muller Juristischen Verlag; trad. it. Sociologia dell'amministrazione pubblica, Bologna, Il Mulino, 1982.

Merton, R.K. (1949), Patterns of Influence: Local, and Cosmopolitan Influentials, in R.K. Merton (a cura di), Social Theory and Social Structure, New York, Free Press; trad. it. Teoria e struttura sociale, 3 voll., Bologna, Il Mulino, 1971.

McGregor, E.G. (1974), Politics and the Career Mobility of Bureaucrats, in «American Political Science Review», LXVIII, pp. 18-26.

Meoli, C. (1984), Il prefetto nell'ordinamento italiano, Firenze, Casa Editrice Noccioli.

Mortara, V. (1989), L'apparato ministeriale: il nucleo centrale della pubblica amministrazione, in G. Freddi (a cura di), La scienza dell'amministrazione, Firenze, Nuova Italia Scientifica, pp. 99-121.

Page, E. (1985), Political Authority and Bureaucratic Power: a Comparative Analysis, Brighton, Wheatsheaf Books; trad. it. Burocrazia, amministrazione, politica, Bologna, Il Mulino, 1990.

Panebianco, A. (1982), Modelli di partito. Potere e organizzazione nei partiti politici, Bologna, Il Mulino.

- (1986), Burocrazie pubbliche, in G. Pasquino (a cura di), Manuale di scienza della politica, Bologna, Il Mulino, pp. 383-430.

- (1988), L'amministrazione e $i$ partiti nei paesi industriali avanzati: introduzione, Archivio ISAP, Milano, Giuffrè, pp. 1623-1643.

- (1989), Organizzazione e potere, in A. Panebianco (a cura di), L'analisi della politica, Bologna, Il Mulino, pp. 255-287.

Putnam, R.D. (1973), Atteggiamenti politici dell'alta burocrazia nell'Europa occidentale, in «Rivista Italiana di Scienza della Politica», 1, pp. $145-186$.

Ridley, F.F. (1979), Government and administration in Western Europe, Oxford, M. Robertson.

- (1983), Career service: a comparative perspective on civil service promotion, in «Public Administration», pp. 179-196.

Rose, R. (1976), The problem of party government, London, Macmillan Press.

Sacco, A.M. e G. Sensale (1986), L'ordinamento del ministero dell' industria, commercio e artigianato, Napoli, Simone.

Self, P. (1972), Administrative Theories and Politics, London, George, Allen and Unwin.

Simon, H.A. (1947), Administrative Bebavior, New York, Macmillan; trad. it. Il comportamento amministrativo, Bologna, Il Mulino, 1958. 
Taradel, A. (1989), Sviluppo storico dell'amministrazione italiana dal 1853 al 1972, in G. Freddi (a cura di), La scienza dell'amministrazione, Roma, Nuova Italia Scientifica, pp. 67-98.

Thompson, J.D. (1967), Organizations in Action, New York, McGrow-Hill; trad. it. L'azione organizzativa, Torino, Isedi, 1988.

Virga, P. (1973), Il pubblico impiego, Milano, Giuffrè.

Zan, S. (1989), Teoria delle organizzazioni e amministrazione pubblica, in G. Freddi (a cura di), La scienza dell'amministrazione, Roma, Nuova Italia Scientifica, pp. 281-311. 